IOS Press

\title{
Editorial
}

\section{Semantic technologies and interoperability in the built environment}

\author{
Pieter Pauwels ${ }^{\mathrm{a},{ }^{*}}$, María Poveda-Villalón ${ }^{\mathrm{b}}$ Álvaro Sicilia ${ }^{\mathrm{c}}$ and Jérôme Euzenat ${ }^{\mathrm{d}}$ \\ ${ }^{a}$ Department of Architecture and Urban Planning, Ghent University, Belgium \\ E-mail:pipauwel.pauwels@ugent.be \\ ${ }^{\mathrm{b}}$ Ontology Engineering Group, Universidad Politécnica de Madrid, Spain \\ E-mail: mpoveda@fi.upm.es \\ ${ }^{\mathrm{c}}$ ARC, La Salle Engineering and Architecture, Ramon Llull University, Spain \\ E-mail: alvaro.sicilia@salle.url.edu \\ ${ }^{\mathrm{d}}$ Univ. Grenoble Alpes, Inria, CNRS, Grenoble INP, LIG, France \\ E-mail: Jerome.Euzenat@inria.fr
}

\begin{abstract}
The built environment consists of plenty of physical assets with which we interact on a daily basis. In order to improve not only our built environment, but also our interaction with that environment, we would benefit a lot from semantic representations of this environment. This not only includes buildings, but also large infrastructure (bridges, tunnels, waterways, underground systems), and geospatial data. With this special issue, an insight is given into the current state of the art in terms of semantic technologies and interoperability in this built environment. This editorial not only summarizes the content of the Special Issue on Semantic Technologies and interoperability in the Built Environment, it also provides a brief overview of the current state of the art in general in terms of standardisation and community efforts.
\end{abstract}

Keywords: Semantics, built environment, building information model, linked data, architecture, construction

\section{Semantics in the built environment}

The built environment encompasses a variety of artifacts ranging from buildings to infrastructures. These artifacts are linked at different scales and in various ways. Namely, relations can be found between elements in a singular building, furniture, street lights, and so forth to elements and concepts covering the entire city, such as infrastructure, traffic, and people flows. Apart from this multiplicity of artifacts, the built environment also gathers multiple stakeholders who collaborate in various ways. Collaboration and interaction not only happens in the built environment as it exists on a daily basis, but even more so throughout

\footnotetext{
*Corresponding author. E-mail: pipauwel.pauwels@ugent.be.
}

all the design, construction and operation phases taking place within the built environment. This includes specialists (architects, engineers, and contractors), but also local administrators, facility managers, and citizens.

A large part of this environment is governed by the Architecture, Engineering, and Construction (AEC) industry. An effective collaboration and interoperability between these different actors throughout the lifecycle of the built environment has always been a key challenge to this industry. Data from stakeholders is modeled and published in various languages and scales, in particular using Building Information Modelling (BIM) tools [2], and data evolves considerably over time. Hence, maintaining data consistency throughout the whole life-cycle of a building, espe- 
cially during the design and construction phases, is a fundamental challenge to this industry.

Beyond the design and construction phases, important other amounts of data are present as well in the built environment in general. This data is used to make the built environment function on a daily basis. Devices are used to retrieve information from the built environment (monitoring systems at the building and urban level) and models of the artifacts interact with multiple tools (simulations of the building energy performance, level of occupancy in buildings) in order to take more informed decisions to improve the built environment. Therefore, flexible mechanisms are required to facilitate data exchange between the different actors intervening at the different stages, and to provide the required interoperability between data, tools and devices.

Standard data models such as the Industry Foundation Classes $\left(\right.$ IFC $^{1}$ ) - for building models - and CityGML - for urban models - support the interoperability between data and tools. However, they often lack the flexibility that is required by working processes, which are, more often than not, unique and difficult to standardize and replicate. For this purpose, web technologies can be used, as they bring a decentralized and peer-to-peer governance model to the data. In particular, Semantic Web technologies are being applied to overcome the limitations of the standard data models by enabling more flexible ways to interlink data from various scales and domains in order to facilitate the interoperability among data, tools, systems and actors that intervene in the creation and use of the built environment. Furthermore, flexible data publication and consumption mechanisms, based on Semantic Web technologies, facilitate the accessibility of data from different scales and domains, such as geographical data (GIS data), urban data (socio-economic, planning), infrastructure data (Smart Grids) and social data (user feedback).

\section{Special issue contributions}

From the 8 papers submitted to the special issue, the following 5 were selected for publication. The papers accepted in this special issue are representative examples of how Semantic Web technologies can be applied to make a better use of data models for the built en-

\footnotetext{
${ }^{1}$ http://www.buildingsmart-tech.org/specifications/ifc-releases/ summary
}

vironment. Two papers focus on the energy efficiency of buildings and cities, namely Esnaola-Gonzalez et al. [3] and Bonino and De Russis [1]. Building home automation is addressed by two papers from a perspective of a social network of agents (Ruta et al. [8]) and an all-seeing smart building (Marroquin et al. [4]). Zhang et al. [9] propose a SPARQL extension for querying building data in IFC.

In DogOnt as a viable seed for semantic modeling of $A E C / F M$, Bonino and De Russis [1] analyze the state of the art of ontologies for electrical and thermal consumption. In this review, authors conclude that current models address each level and domain separately, therefore preventing the representation of the energy domain in a structured and comprehensive way. After that, the paper presents in detail the latest DogOnt ontology (version 4.0), which can be used as a foundation for a shared and unified view of energy profiles at different levels. The design choices and adaptations made form the first DogOnt released in 2008 are discussed, showing how the model is up to date and increasingly adopted for the energy representation in the Smart Cities domain.

Esnaola-Gonzalez et al. [3] present in their paper a Semantic prediction assistant approach applied to energy efficiency in tertiary buildings. They argue how fulfilling occupants' comfort whilst reducing energy consumption is still an unsolved problem in most of tertiary buildings, even though the expansion of the Internet of Things (IoT) and Knowledge Discovery in Databases (KDD) techniques may allow to improve matters in this regard. In their paper, therefore, they propose the EEPSA (Energy Efficiency Prediction Semantic Assistant) process, which leverages Semantic Web technologies to enhance the KDD process for achieving energy efficiency in tertiary buildings while maintaining comfort levels. This process guides the data analyst through the different KDD phases in a semi-automatic manner and supports Heating, Ventilation, and Air-Conditioning (HVAC) system activation strategies. That is, temperature of a space is predicted simulating the activation of HVAC systems at different times and intensities, so that the facility manager can choose the strategy that best fits both the user's comfort needs and energy efficiency.

The paper Ontology for a Panoptes building: Exploiting contextual information and a smart camera network by Marroquin et al. [4] proposes the WiseNET ontology for a Panoptes building, which integrates data from smart camera networks to monitor different activities that occur in the built environment and contextual 
IFC data to enable real-time event detections and system reconfiguration. The contributions of the authors overcome the multi-camera based system limitations and some computer vision problems such as missed detections, false detections, and occlusions. The use of semantic knowledge in multi-camera monitoring systems guarantees the protection of the user privacy by not sending nor saving any image, just extracting the knowledge from them.

In Social Internet of things for domotics: A knowledge-based approach over LDP-CoAP, Ruta et al. [8] describe a novel semantic-based framework complying with the emerging Social Internet of Things for home and building automation. Device agents, placed in building spaces, are organized in social networks, interacting autonomously and sharing information, cooperating and orchestrating resources. In particular, the authors use the Semantic Web languages as a knowledge representation layer, and propose mobileoriented implementations of non-monotonic inferences for semantic matchmaking used to give decision capabilities to software agents. The Linked Data Platform (LDP) is used with the Constrained Application Protocol (CoAP) to provide the knowledge organization and sharing infrastructure underpinning social object interactions.

In BimSPARQL: Domain-specific functional SPARQL extensions for querying RDF building data, Zhang et al. [9] propose an extension of SPARQL functions in order to query IFC building data. The presented approach is driven by the IFC documentation and BIM requirement checking use cases. The goal of the proposed extension is two-fold: 1) to simplify writing queries, and 2) to retrieve useful information implied in 3D geometry data according to requirement checking use cases. RDF vocabularies are used to model the extended functions which are implemented by combining declarative rules with procedural programming. Finally, authors discuss the approach applicability and advantages as well as current issues and future challenges.

\section{Semantics and linked data for the built environment}

As is clear from the papers published in this Special Issue, the built environment deals with lots of semantics, of various kinds and covering many domains. Because of the wide range of stakeholders involved in the built environment, especially in design and construc- tion phases, standards and agreements and processes are of utmost importance, especially to the AEC industry. One of the most important standards in this regard is the IFC data model, as produced by buildingSMART. This data model consists of hundreds of entities and is defined using the EXPRESS information modelling language. Furthermore, there is a strong focus on the representation of $3 \mathrm{D}$ geometry. Notwithstanding the existence of an ifcOWL ontology [5], IFC is not so easily accessible or usable using Semantic Web technologies. This low usability is mostly due to the strong reliance on EXPRESS, the large size and complexity of the data model and the non-modular structure of IFC.

A number of other ontologies and data models have been produced in the last few years, mainly under the auspices of the World Wide Web Consortium (W3C), aiming to enable the representation of the built environment in a Semantic Web-friendly manner. This includes the ontologies that are produced in the W3C Linked Building Data (LBD) Community Group. ${ }^{2}$ These ontologies are inspired by the IFC data model, but start from a simpler and more modular structure. Ontologies include the Building Topology Ontology (BOT [7]), a set of PRODUCT ontologies, and PROPS ontologies. The latter two ontologies mainly aim at allowing the representation of building elements (walls, windows, columns, etc.) and their properties (fire resistance, thermal performance, etc.).

Starting from this modular structure, it is easier to connect semantic representations of buildings with semantic representations of the rest of the built environment, thereby linking to many of the ontologies and topics that are covered by this Special Issue, namely DogOnt, EEPSA, SAREF, SOSA, SSN, CityGML, GeoSPARQL, and so on. As an example, many of the work behind the included article about BimSPARQL [9] is inspired by the GeoSPARQL principles. Using these ontologies, plenty applications are within reach, based on Semantic Web technologies, which have been unaccessible for many stakeholders in the built environment, including (1) the direct combination of sensor data with building element information, (2) the combination of geospatial and building data, and (3) real-time event detection and then analysis of building use patterns.

Many of the contributed papers and the above efforts align with the three main advantages of semantic web

\footnotetext{
${ }^{2}$ https://www.w3.org/community/lbd/
} 
technologies to the AEC domain that were outlined by Pauwels et al. [6]: interoperability, linking across domains, and logical inference and proofs. As indicated by Pauwels et al. [6], interoperability requires standardization more than it requires the use of one or the other technology. Therefore, semantic web technologies have only a minor contribution there. In terms of linking data and domains, it is clear from this special issue and all the above developments that this is a main contribution of relying on semantic web technologies. Finally, logical inference and proof is less present in the special issue, even though this is a unique feature offered by semantic web technologies.

In conclusion, Semantic Web technologies allow communities to build a decentralized and easily extensible semantic network in a modular fashion for the built environment. This allows a global community of developers to build a myriad of applications that were not feasible or available before on such a scale, covering not only the AEC domain, but also topics and ventures like Smart Cities, semantic sensor networks, home automation systems, smart grids, and geographical information systems.

\section{References}

[1] D. Bonino and L. De Russis, DogOnt as a viable seed for semantic modeling of AEC/FM, Semantic Web 9(6) (2018), 763-780.
[2] C. Eastman, P. Teicholz, R. Sacks and K. Liston, BIM Handbook: A Guide to Building Information Modeling for Owners, Managers, Designers, Engineers, and Contractors, 2nd edn, Wiley, Hoboken NJ, USA, 2008. ISBN 9780470185285.

[3] I. Esnaola-Gonzalez, J. Bermúdez, I. Fernandez and A. Arnaiz, Semantic prediction assistant approach applied to energy efficiency in tertiary buildings, Semantic Web 9(6) (2018), 735-762.

[4] R. Marroquin, J. Dubois and C. Nicolle, Ontology for a Panoptes building: Exploiting contextual information and a smart camera network, Semantic Web 9(6) (2018), 803-828.

[5] P. Pauwels and W. Terkaj, EXPRESS to OWL for construction industry: Towards a recommendable and usable ifcOWL ontology, Automation in Construction 63 (2016), 100-133. doi:10. 1016/j.autcon.2015.12.003.

[6] P. Pauwels, S. Zhang and Y.-C. Lee, Semantic web technologies in AEC industry: A literature overview, Automation in Construction 73 (2017), 145-165. doi:10.1016/j.autcon.2016. 10.003 .

[7] M.H. Rasmussen, P. Pauwels, C.A. Hviid and J. Karlshoj, Proposing a central AEC ontology that allows for domain specific extensions, in: Lean and Computing in Construction Congress (LC3): Volume I-Proceedings of the Joint Conference on Computing in Construction (JC3), F. Bossché, I. Brilakis and R. Sacks, eds, Heraklion, Vol. 1, Crete, Greece, 2017, pp. 237244. doi:10.24928/JC3-2017/0153.

[8] M. Ruta, F. Scioscia, G. Loseto, F. Gramegna, S. Ieva, A. Pinto and E.D. Sciascio, Social internet of things for domotics: A knowledge-based approach over LDP-CoAP, Semantic Web 9(6) (2018), 781-802.

[9] C. Zhang, J. Beetz and B. de Vries, BimSPARQL: Domainspecific functional SPARQL extensions for querying RDF building data, Semantic Web 9(6) (2018), 829-855. 